\title{
Regeneração de uma Floresta Ombrófila Mista no Planalto Catarinense
}

\author{
João Paulo de Maçaneiro ${ }^{1 *}$ \\ Rafaela Cristina Seubert ${ }^{2}$ \\ Allisson Heilmann ${ }^{2}$ \\ Lauri Amândio Schorn ${ }^{2}$ \\ ${ }^{1}$ Universidade Federal do Paraná, Setor de Ciências Agrárias \\ Departamento de Ciências Florestais \\ Avenida Prefeito Lothário Meissner, 900, Jardim Botânico, CEP 80210-170, Curitiba - PR, Brasil \\ ${ }^{2}$ Universidade Regional de Blumenau, Centro de Ciências Tecnológicas \\ Departamento de Engenharia Florestal \\ Rua São Paulo, 3250, Itoupava Seca, CEP 89010-971, Blumenau - SC, Brasil \\ * Autor para correspondência \\ jpmacaneiro@gmail.com
}

Submetido em 17/03/2016

Aceito para publicação em 07/10/2016

\section{Resumo}

Estudos sobre regeneração florestal podem fornecer informações sobre as espécies que irão compor a floresta no futuro. Entretanto, poucos estudos enfatizam esse componente. Neste trabalho, avaliou-se a regeneração arbórea e arbustiva de uma Floresta Ombrófila Mista em 180 parcelas de 2,5 m de raio, onde foram medidos os indivíduos com altura $\geq 50 \mathrm{~cm}$ e DAP $\leq 5 \mathrm{~cm}$. Foram amostrados 4.078 indivíduos e 89 espécies. Os índices de Shannon $(3,64)$ e Pielou $(0,81)$ evidenciaram alta diversidade e equilíbrio na abundância das espécies. As espécies mais importantes da regeneração foram Allophylus edulis, Casearia decandra, Cupania vernalis, Matayba elaeagnoides e Myrsine coriacea. Os resultados mostram que nem todas as espécies mais importantes apresentam tendência à forma de J-invertido, evidenciando que pode estar havendo desequilíbrio entre as taxas de mortalidade e recrutamento dos indivíduos dessas espécies na regeneração.

Palavras-chave: Distribuição de alturas; Fitossociologia; Floresta de Araucária; Regeneração florestal; Riqueza de espécies

\section{Abstract}

Regeneration of a Mixed Ombrophilous Forest on the Santa Catarina Plateau. Studies on forest regeneration can provide information about species that will form a forest in the future. However, few studies emphasize this component. This study evaluated tree and shrub regeneration in a Mixed Ombrophilous Forest using 180 sample plots with $2.5 \mathrm{~m}$ radius, where individuals whose height was $\geq 50 \mathrm{~cm}$ and diameter at breast height (DBH) was $\leq 5.0 \mathrm{~cm}$. The sample consisted of 4,078 individuals and 89 species. The Shannon index (3.64) and the Pielou index (0.81) showed a high diversity and balance regarding the abundance of 
species. The most significant species for regeneration were Allophylus edulis, Casearia decandra, Cupania vernalis, Matayba elaeagnoides, and Myrsine coriacea. The results show that not all of the most important species tend exhibit an inverted $\mathrm{J}$ shape, indicating the possibility of imbalance between the mortality and recruitment rates of these species in regeneration.

Key words: Araucaria forest; Forest regeneration; Height distribution; Phytosociology; Species richness and diversity

\section{Introdução}

O domínio da Mata Atlântica constitui uma região heterogênea, com diferentes fitofisionomias e elevada riqueza de espécies que por vezes superam a das florestas amazônicas (THOMAS et al., 1998). Entre as fitofisionomias com maior riqueza de espécies da Mata Atlântica está a Floresta Ombrófila Mista (Floresta de Araucária) (LEITE, 2002; IBGE, 2012), caracterizada pela mistura das floras tropical (afro-brasileira) e temperada (austral-antártica-andina), com destaque para os elementos Coniferales (Araucaria e Podocarpus) e Laurales (Ocotea, Nectandra e Cryptocarya) (LEITE, 2002; KERSTEN et al., 2015).

Em Santa Catarina, a Floresta Ombrófila Mista compreende grande parte das formações do Planalto Meridional e está dividida em Floresta Aluvial, Submontana, Montana e Alto-montana (IBGE, 2012). Originalmente, essa fitofisionomia ocupava cerca de $43.000 \mathrm{~km}^{2}$ da superfície do estado de Santa Catarina, mas, devido ao intenso processo de exploração de seus recursos, se encontra reduzida a apenas $12.317 \mathrm{~km}^{2}$ (VIBRANS et al., 2013). Atualmente, grande parte dessas florestas está constituída por fragmentos com fisionomia de vegetação secundária em estágios médio ou avançado de regeneração, sendo raros os remanescentes com florestas primárias (SEVEGNANI et al., 2013).

Em florestas secundárias, a regeneração florestal pode ser entendida como um processo de sucessão secundária em nível de comunidade e de ecossistema, sobre uma área desmatada e que anteriormente apresentava floresta (CHAZDON, 2012). As florestas em processo de regeneração podem constituir fontes essenciais de produtos madeireiros e não madeireiros, além de oferecerem carbono atmosférico (PAN et al., 2011) e habitats adequados para diferentes espécies da fauna e flora regional (DENT; WRIGHT, 2009). Em
Santa Catarina, poucos estudos avaliaram os estágios sucessionais da Floresta Ombrófila Mista (KLEIN, 1960; SEVEGNANI et al., 2013; SIMINSKI et al., 2013), com destaque para os de Klein (1960), o qual verificou que a regeneração florestal variava de acordo com o grau de umidade dos solos. Assim, esse autor definiu duas trajetórias sucessionais de regeneração: a primeira ocorria em solos muito úmidos, onde a vegetação poderia apresentar até sete associações vegetais com composição de espécies distintas, e a segunda ocorria nos campos limpos com solos mais secos, onde Araucaria angustifolia era a principal espécie a colonizar a área e modificar as condições ambientais. Além disso, as trajetórias sucessionais de regeneração dessas florestas podem ocorrer em diferentes escalas e variam de acordo com o tipo do uso anterior do solo, proximidade da área com remanescentes florestais e presença da fauna dispersora regional, entre outros (CHAZDON, 2012).

O estudo da regeneração de florestas pode auxiliar no entendimento dos processos sucessionais e de manutenção da diversidade de espécies, pela comparação da composição, estrutura e dinâmica da regeneração e das plantas adultas ao longo do tempo (FELFILI, 1997; CHAZDON, 2012; SIMINSKI et al., 2013). Aliado a isso, é possível fornecer informações sobre as trajetórias sucessionais, permitindo identificar quais espécies que irão compor a floresta no futuro e quais espécies são aptas para uso em projetos de restauração (SILVA et al., 2013). Além disso, os padrões de crescimento e regeneração das espécies podem servir de base para distinguir florestas secundárias antigas de florestas intocadas pelo homem (CHAZDON, 2012).

Nos últimos anos, em função da atual situação de degradação da Floresta Ombrófila Mista em Santa Catarina, alguns estudos foram realizados com o propósito de caracterizar a composição e estrutura de fragmentos florestais. Entretanto, grande parte desses 
estudos enfatizou o estrato arbóreo da vegetação (KLAUBERG et al., 2010; VIBRANS et al., 2011; HIGUCHI et al., 2012; SILVA et al., 2012b; 2013), sendo poucos os estudos voltados à regeneração florestal (FIORENTIN et al., 2015; HIGUCHI et al., 2015). Apesar de a maioria desses estudos terem enfatizado o estrato arbóreo, eles servem de base para determinar padrões florísticos em estudos de meta-análise (SILVA et al., 2013) e a análise de vários estudos que enfatizam a regeneração de florestas pode servir de base na definição de trajetórias sucessionais e na escolha de espécies para projetos de restauração, além de possibilitarem a geração de novas hipóteses sobre a relação entre as espécies e as condições ambientais, as quais não seriam possíveis sem a soma de vários estudos já publicados (MAÇANEIRO et al., 2015b). Neste sentido, para caracterizar a regeneração natural de um remanescente de Floresta Ombrófila Mista no Planalto de Santa Catarina, o presente trabalho teve como objetivo obter informações sobre a composição florística e estrutura fitossociológica de indivíduos arbóreos e arbustivos regenerantes, de modo a fornecer informações básicas para ações de conservação e restauração dessas florestas.

\section{Material e Métodos}

A área de estudo está inserida na bacia hidrográfica do rio Pelotas, em Campo Belo do Sul, Santa Catarina. Pertence à RPPN Emílio Einsfeld Filho, que possui área total de $6.328,6$ ha, altitude que varia de 620 a 980 m e está localizada entre $27^{\circ} 58^{\prime}$ e $28^{\circ} 04^{\prime} \mathrm{S}$ e $50^{\circ} 54^{\prime}$ e $50^{\circ} 45^{\prime} \mathrm{W}$ (Figura 1). O clima da região de estudo, segundo classificação de Köppen, é do tipo $\mathrm{Cfb}$ - clima temperado mesotérmico úmido com verão ameno. A temperatura média anual varia entre $16-17^{\circ} \mathrm{C}$, com umidade relativa média anual entre $78-80 \%$ e precipitação total anual de $1.527 \mathrm{~mm}$, bem distribuída durante o ano (EPAGRI, 2002).

A geologia da região é formada pelo Grupo São Bento, composto pela Formação Serra Geral, que é constituída por intrusões de basaltos, diabásios e lavas ácidas (SANTA CATARINA, 1986). Predomina, na

FIGURA 1: Localização das 180 parcelas utilizadas para amostrar as espécies da regeneração de uma Floresta Ombrófila Mista, RPPN Emílio Einsfeld Filho, Santa Catarina.

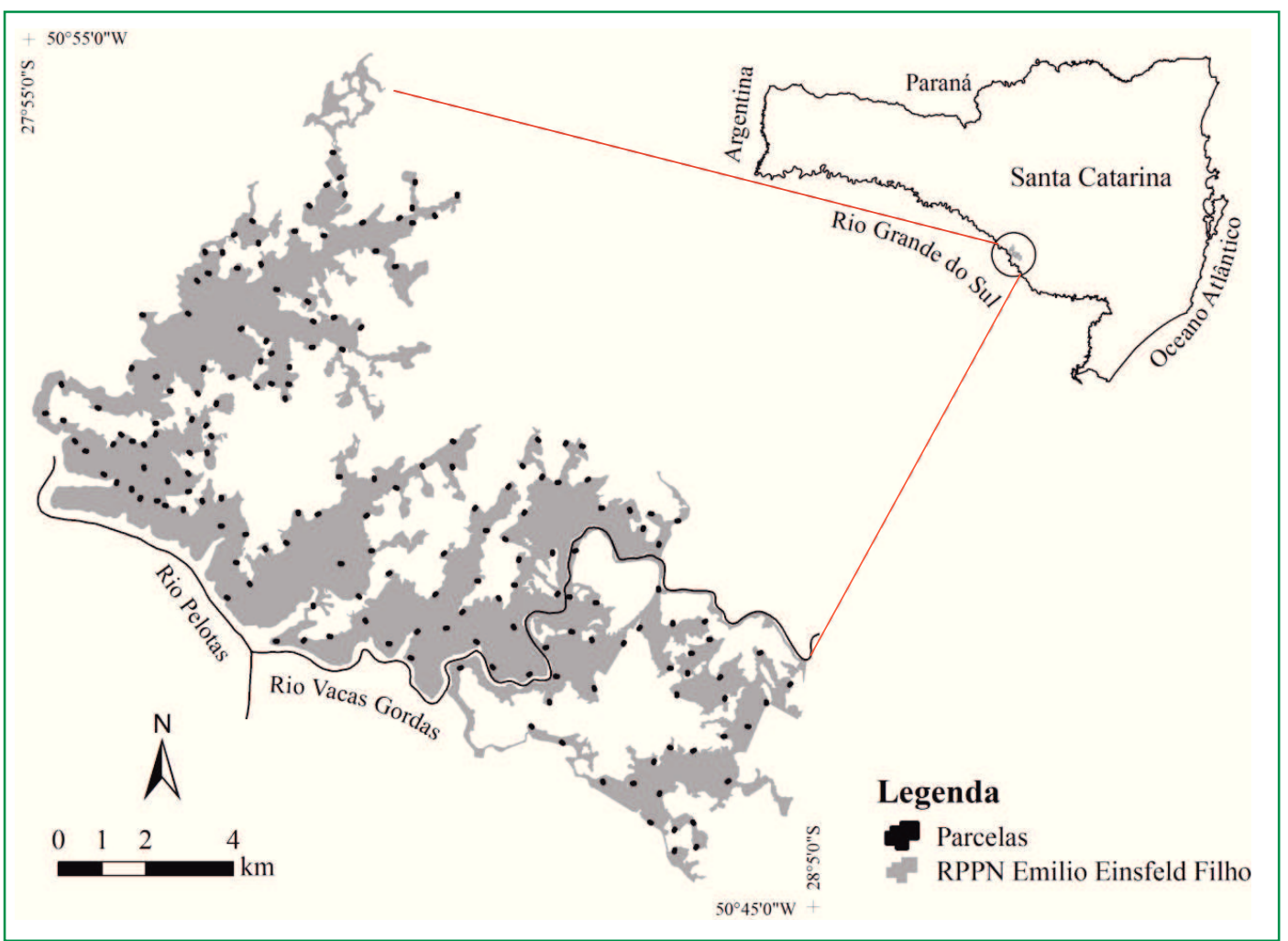


região de estudo, uma associação entre dois tipos de solo: em relevos forte ondulados, o Neossolo Litólico Álico A proeminente de textura argilosa e, em relevos ondulados, o Cambissolo Álico $\mathrm{Tb}$ A proeminente de textura argilosa (EMBRAPA, 2004).

A vegetação da área de estudo é constituída pela Floresta Ombrófila Mista Montana (IBGE, 2012), caracterizada pelos densos agrupamentos formados por Araucaria angustifolia (Bertol.) Kuntze (pinheirobrasileiro), entremeados por campos naturais (LEITE, 2002). A RPPN Emílio Einsfeld Filho foi alvo de exploração madeireira até 1985. Essa exploração teve como foco o pinheiro-brasileiro e outras espécies de interesse econômico. Atualmente, o estado de conservação da floresta na área de estudo pode ser classificado como vegetação em estágio avançado de regeneração, com cerca de 30 anos sem exploração antrópica.

Para amostrar a regeneração natural foram distribuídas de forma aleatória 180 parcelas circulares com 2,5 m de raio, totalizando 3.534,30 $\mathrm{m}^{2}$ de área amostral (Figura 1). Essas parcelas foram aleatorizadas e inseridas próximas à borda da floresta, em uma distância mínima de $20 \mathrm{~m}$. Em cada parcela foram registrados e identificados todos os indivíduos arbóreos e arbustivos vivos, incluindo palmeiras e fetos arborescentes, com altura $\geq 50 \mathrm{~cm}$ e perímetro à altura do peito $(\mathrm{PAP}) \leq 15 \mathrm{~cm}$.

O material botânico coletado foi identificado por comparação com exsicatas depositadas nos Herbários do Laboratório de Dendrologia e Dr. Roberto Miguel Klein, da Universidade Regional de Blumenau (FURB), e por meio de consulta à literatura taxonômica e aos especialistas da FURB. As espécies foram agrupadas em famílias, seguindo o sistema proposto pelo Angiosperm Phylogeny Group IV (APG IV, 2016) e utilizando a base de dados da Flora do Brasil (2016).

A riqueza de espécies observadas na regeneração foi analisada por meio da rarefação pelo método Mao Tau e baseada no número de parcelas, sendo que a riqueza de espécies foi estimada a partir do estimador Chao2, e a métrica diversidade de espécies calculada a partir do índice de Shannon (H', base-ln) e do índice de Pielou (MAGURRAN, 2004).

Para analisar a estrutura da regeneração foram calculados os parâmetros fitossociológicos: densidade, frequência e classes de tamanho absolutas e relativas e o valor de regeneração natural para cada espécie (HOSOKAWA et al., 2008). Em seguida, as espécies foram classificadas por grupo ecológico, adotando-se a metodologia de Swaine e Whitmore (1988) e OliveiraFilho (1994) nas seguintes categorias: pioneira (P), clímax exigente de luz (CL) e clímax tolerante à sombra (CS).

Os indivíduos regenerantes foram agrupados em nove classes de altura, todas com amplitudes de 1,0 m. Com o intuito de averiguar semelhanças ou diferenças entre a distribuição de alturas da regeneração com as cinco espécies mais importantes, foram utilizados o teste de Kolmogorov-Smirnov para duas amostras ao nível de significância $\alpha=0,05$ (SIEGEL; CASTELLAN, 2006) e o coeficiente de Gini (WEINER; SOLBRIG, 1984).

\section{Resultados}

A riqueza amostrada foi de 89 espécies e o estimador não paramétrico Chao2 estimou uma riqueza total de 102 espécies para a área de estudo (Figura 2). O índice de diversidade de Shannon (H', base- $l n$ ) para a área de estudo foi de 3,64 e o índice de Pielou (J') foi de 0,81.

FIGURA 2: Curva de rarefação (Mao Tau) e estimador de riqueza (Chao2) para as 180 parcelas da regeneração natural em uma Floresta Ombrófila Mista, Santa Catarina.

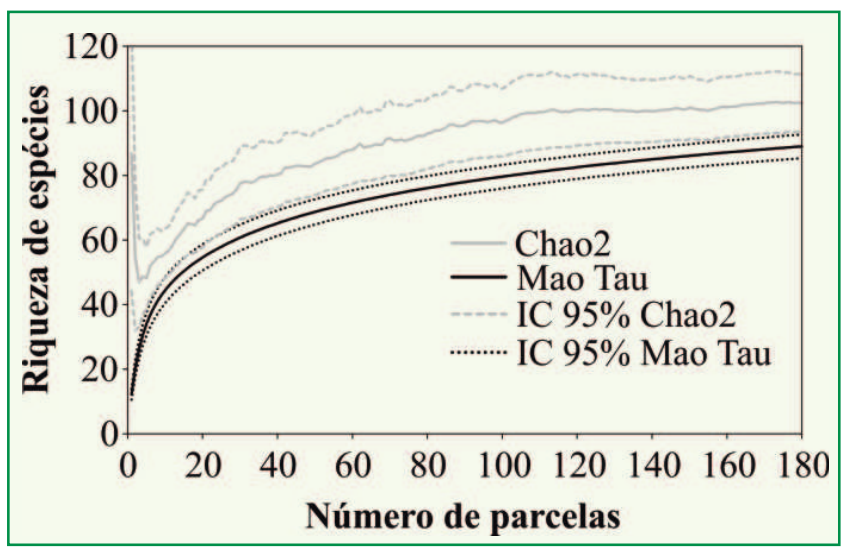

No total, foram amostrados 4.078 indivíduos pertencentes a 66 gêneros e 40 famílias (Tabela 1). Entre as espécies amostradas, 85 (95,5\%) foram identificadas em nível de espécie e apenas quatro (4,5\%) em nível de gênero. Foi registrada também uma espécie exótica: Aleurites moluccanus (L.) Willd. 
TABELA 1: Famílias e espécies registradas na regeneração natural em uma Floresta Ombrófila Mista, Santa Catarina. $\mathrm{N}$ = número de indivíduos; $\mathrm{GE}=$ grupo ecológico, $\mathrm{P}=$ pioneira, $\mathrm{CL}=$ clímax exigente de luz, $\mathrm{CS}=$ clímax tolerante à sombra.

\begin{tabular}{|c|c|c|c|}
\hline Família & Espécie & $\mathbf{N}$ & GE \\
\hline Anacardiaceae & Schinus terebinthifolius Raddi & 1 & $\mathrm{P}$ \\
\hline Annonaceae & Annona emarginata (Schltdl.) H.Rainer & 87 & CL \\
\hline Apocynaceae & Aspidosperma tomentosum Mart. & 1 & $\mathrm{CS}$ \\
\hline Aquifoliaceae & Ilex theezans Mart. & 25 & CL \\
\hline Araucariaceae & Araucaria angustifolia (Bertol.) Kuntze & 76 & $\mathrm{CL}$ \\
\hline Arecaceae & Syagrus romanzoffiana (Cham.) Glassman & 12 & $\mathrm{CL}$ \\
\hline \multirow[t]{3}{*}{ Asteraceae } & Gochnatia polymorpha (Less.) Cabrera & 1 & $P$ \\
\hline & Piptocarpha angustifolia Dusén & 1 & $\mathrm{P}$ \\
\hline & Vernonanthura discolor (Spreng.) H.Rob. & 3 & $\mathrm{P}$ \\
\hline \multirow[t]{2}{*}{ Bignoniaceae } & Handroanthus catarinensis (A.H.Gentry) S.O.Grose & 1 & CL \\
\hline & Jacaranda micrantha Cham. & 2 & $\mathrm{CL}$ \\
\hline Boraginaceae & Cordia americana (L.) Gottschling \& J.S.Mill. & 2 & $\mathrm{CL}$ \\
\hline Canellaceae & Cinnamodendron dinisii Schwacke & 75 & CL \\
\hline Cardiopteridaceae & Citronella gongonha (Mart.) R.A.Howard & 8 & $\mathrm{CL}$ \\
\hline Celastraceae & Maytenus aquifolia Mart. & 9 & CS \\
\hline Clethraceae & Clethra scabra Pers. & 2 & $\mathrm{P}$ \\
\hline \multirow[t]{2}{*}{ Cunoniaceae } & Lamanonia ternata Vell. & 4 & CL \\
\hline & Weinmannia humilis Engl. & 1 & $\mathrm{CL}$ \\
\hline Cyatheaceae & Alsophila setosa Kaulf. & 30 & CS \\
\hline Dicksoniaceae & Dicksonia sellowiana Hook. & 25 & $\mathrm{CS}$ \\
\hline Erythroxylaceae & Erythroxylum sp. & 2 & $\mathrm{CL}$ \\
\hline Escalloniaceae & Escallonia bifida Link \& Otto & 1 & $\mathrm{P}$ \\
\hline \multirow[t]{5}{*}{ Euphorbiaceae } & *Aleurites moluccanus (L.) Willd & 1 & - \\
\hline & Bernardia pulchella (Baill.) Müll.Arg. & 9 & $\mathrm{CL}$ \\
\hline & Gymnanthes klotzschiana Müll.Arg. & 104 & $P$ \\
\hline & Sapium glandulosum (L.) Morong & 19 & $\mathrm{CL}$ \\
\hline & Sebastiania brasiliensis Spreng. & 185 & $\mathrm{CL}$ \\
\hline \multirow[t]{7}{*}{ Fabaceae } & Bauhinia forficata Link & 3 & CL \\
\hline & Dalbergia frutescens (Vell.) Britton & 22 & $\mathrm{CL}$ \\
\hline & Inga virescens Benth. & 14 & CS \\
\hline & Lonchocarpus campestris Benth. & 43 & $\mathrm{CL}$ \\
\hline & Mimosa scabrella Benth. & 2 & $\mathrm{P}$ \\
\hline & Myrocarpus frondosus Allemão & 11 & $\mathrm{CL}$ \\
\hline & Parapiptadenia rigida (Benth.) Brenan & 19 & CL \\
\hline \multirow[t]{5}{*}{ Lauraceae } & Nectandra grandiflora Nees & 3 & CS \\
\hline & Nectandra lanceolata Nees & 25 & CS \\
\hline & Nectandra megapotamica (Spreng.) Mez & 170 & $\mathrm{CL}$ \\
\hline & Ocotea puberula (Rich.) Nees & 12 & $\mathrm{CL}$ \\
\hline & Ocotea pulchella (Nees \& Mart.) Mez & 156 & $\mathrm{CL}$ \\
\hline Loganiaceae & Strychnos brasiliensis Mart. & 33 & CS \\
\hline Malvaceae & Luehea divaricata Mart. \& Zucc. & 12 & $\mathrm{P}$ \\
\hline Melastomataceae & Miconia cinerascens Miq. & 13 & CS? \\
\hline Meliaceae & Cedrela fissilis Vell. & 2 & $\mathrm{CL}$ \\
\hline \multirow[t]{4}{*}{ Myrtaceae } & Blepharocalyx salicifolius (Kunth) O.Berg & 53 & $\mathrm{CL}$ \\
\hline & Calyptranthes concinna DC. & 91 & CS \\
\hline & Campomanesia guaviroba (DC.) Kiaersk. & 54 & CS \\
\hline & Campomanesia guazumifolia (Cambess.) O.Berg & 1 & CS \\
\hline
\end{tabular}




\begin{tabular}{|c|c|c|c|}
\hline & Eugenia multicostata D.Legrand & 75 & $\mathrm{CS}$ \\
\hline & Eugenia pyriformis Cambess. & 112 & CS \\
\hline & Eugenia rostrifolia D.Legrand & 62 & CS \\
\hline & Eugenia sp. & 116 & - \\
\hline & Eugenia uniflora L. & 78 & CS \\
\hline & Myrceugenia sp. & 105 & CS \\
\hline & Myrcia hatschbachii D.Legrand & 1 & $\mathrm{CS}$ \\
\hline & Myrcia palustris DC. & 52 & CL \\
\hline & Myrcia selloi (Spreng.) N.Silveira & 1 & CS \\
\hline & Myrcia sp. & 56 & - \\
\hline & Myrcianthes pungens (O.Berg) D.Legrand & 53 & CS \\
\hline Peraceae & Pera glabrata (Schott) Poepp. ex Baill. & 6 & $\mathrm{CL}$ \\
\hline Phytolaccaceae & Seguieria aculeata Jacq. & 7 & $\mathrm{CL}$ \\
\hline Podocarpaceae & Podocarpus lambertii Klotzsch ex Endl. & 17 & CS \\
\hline \multirow[t]{3}{*}{ Primulaceae } & Myrsine coriacea (Sw.) R.Br. ex Roem. \& Schult. & 197 & $\mathrm{P}$ \\
\hline & Myrsine lancifolia Mart. & 2 & $\mathrm{CL}$ \\
\hline & Myrsine umbellata Mart. & 4 & $\mathrm{CL}$ \\
\hline Proteaceae & Roupala montana Aubl. & 23 & CL \\
\hline \multirow[t]{2}{*}{ Rosaceae } & Prunus brasiliensis (Cham. \& Schltdl.) D.Dietr. & 4 & $\mathrm{CL}$ \\
\hline & Prunus myrtifolia (L.) Urb. & 39 & $\mathrm{CL}$ \\
\hline Rubiaceae & Rudgea parquioides (Cham.) Müll.Arg. & 86 & CS \\
\hline \multirow[t]{4}{*}{ Rutaceae } & Helietta apiculata Benth. & 28 & $\mathrm{CL}$ \\
\hline & Zanthoxylum kleinii (R.S.Cowan) P.G.Waterman & 3 & CL \\
\hline & Zanthoxylum petiolare A.St.-Hil. \& Tul. & 1 & $\mathrm{CL}$ \\
\hline & Zanthoxylum rhoifolium Lam. & 6 & $\mathrm{CL}$ \\
\hline \multirow[t]{4}{*}{ Salicaceae } & Casearia decandra Jacq. & 304 & $\mathrm{CL}$ \\
\hline & Casearia obliqua Spreng. & 30 & $\mathrm{CL}$ \\
\hline & Casearia sylvestris $\mathrm{Sw}$. & 2 & $\mathrm{CL}$ \\
\hline & Xylosma ciliatifolia (Clos) Eichler & 21 & $\mathrm{CL}$ \\
\hline \multirow[t]{5}{*}{ Sapindaceae } & Allophylus edulis (A.St.-Hil. et al.) Hieron. ex Niederl. & 391 & $\mathrm{CL}$ \\
\hline & Allophylus guaraniticus (A.St.-Hil.) Radlk. & 11 & $\mathrm{CL}$ \\
\hline & Allophylus puberulus (Cambess.) Radlk. & 175 & $\mathrm{CL}$ \\
\hline & Cupania vernalis Cambess. & 237 & $\mathrm{CL}$ \\
\hline & Matayba elaeagnoides Radlk. & 188 & $\mathrm{CL}$ \\
\hline \multirow[t]{5}{*}{ Solanaceae } & Brunfelsia pilosa Plowman & 37 & $\mathrm{CL}$ \\
\hline & Capsicum flexuosum Sendtn. & 11 & $\mathrm{CL}$ \\
\hline & Solanum mauritianum Scop. & 1 & $\mathrm{P}$ \\
\hline & Solanum sanctae-catharinae Dunal & 6 & $\mathrm{P}$ \\
\hline & Solanum sp. & 1 & - \\
\hline Styracaceae & Styrax leprosus Hook. \& Arn. & 96 & CL \\
\hline Thymelaeaceae & Daphnopsis racemosa Griseb. & 3 & $\mathrm{CL}$ \\
\hline Winteraceae & Drimys brasiliensis Miers & 4 & $\mathrm{CL}$ \\
\hline
\end{tabular}

* espécie exótica 
As famílias com maior riqueza de espécies foram Myrtaceae (15 espécies), Fabaceae (sete), Euphorbiaceae, Sapindaceae e Solanaceae (cinco cada), com destaque para os gêneros com maior número de espécies: Eugenia (cinco), Myrcia (quatro), Allophylus, Casearia, Myrsine, Nectandra e Zanthoxylum (três cada).

O grupo ecológico mais representativo foi o das espécies clímax exigentes de luz, que corresponderam a 51 espécies e 2.684 indivíduos $(65,8 \%)$, seguido das clímax tolerantes à sombra (21 espécies e 784 indivíduos - 19,2\%) e das pioneiras (12 espécies e 331 indivíduos $8,1 \%$ ). A densidade de indivíduos foi de 11.538 ind.ha $^{-1}$ e as espécies mais abundantes foram Allophylus edulis, Casearia decandra, Cupania vernalis, Myrsine coriacea e Matayba elaeagnoides, as quais somaram $32,3 \%$ da densidade total (Tabela 2).

A frequência absoluta foi relativamente baixa para a maioria das espécies. Apenas quatro espécies (4,5\%) apresentaram frequência superior a 50\%; com frequência entre 20 e 50\%, foram registradas 21 espécies $(23,6 \%)$, enquanto 64 espécies $(71,9 \%)$ apresentaram frequência inferior a $20 \%$. Em relação à estrutura vertical, Allophylus edulis, Cupania vernalis, Casearia decandra e Matayba elaeagnoides se destacaram pelo maior número de indivíduos entre as classes de altura analisadas, correspondendo a $28,2 \%$ da porcentagem total. Essas espécies, junto com Myrsine coriacea, foram as mais importantes da regeneração natural da área de estudo $(\mathrm{RNR}=30 \%)$.

TABELA 2: Parâmetros fitossociológicos calculados para as 20 espécies mais importantes da regeneração natural em uma Floresta Ombrófila Mista, Santa Catarina. DA = densidade absoluta (ind.ha $\left.{ }^{-1}\right)$; DR = densidade relativa (\%); $\mathrm{FA}=$ frequência absoluta $(\%) ; \mathrm{FR}=$ frequência relativa $(\%) ; \mathrm{CAT}=$ classe absoluta de tamanho; $\mathrm{CRT}=$ classe relativa de tamanho (\%); RNR = regeneração natural relativa $(\%)$.

\begin{tabular}{lccccccc}
\hline Espécie & DA & DR & FA & FR & CAT & CRT & RNR \\
\hline Allophylus edulis & 1.106 & 9,6 & 72,2 & 6,1 & 163 & 9,5 & 8,4 \\
Casearia decandra & 860 & 7,5 & 66,7 & 5,6 & 102 & 5,9 & 6,3 \\
Cupania vernalis & 671 & 5,8 & 57,2 & 4,8 & 117 & 6,8 & 5,8 \\
Matayba elaeagnoides & 532 & 4,6 & 53,3 & 4,5 & 101 & 5,9 & 5,0 \\
Myrsine coriacea & 557 & 4,8 & 47,8 & 4,0 & 78 & 4,6 & 4,5 \\
Ocotea pulchella & 441 & 3,8 & 41,1 & 3,5 & 72 & 4,2 & 3,8 \\
Allophylus puberulus & 495 & 4,3 & 34,4 & 2,9 & 71 & 4,1 & 3,8 \\
Sebastiania brasiliensis & 523 & 4,5 & 40,6 & 3,4 & 56 & 3,3 & 3,7 \\
Nectandra megapotamica & 481 & 4,2 & 36,7 & 3,1 & 67 & 3,9 & 3,7 \\
Eugenia pyriformis & 317 & 2,7 & 43,9 & 3,7 & 47 & 2,7 & 3,1 \\
Eugenia sp. & 297 & 2,6 & 33,9 & 2,9 & 49 & 2,9 & 2,8 \\
Myrceugenia sp. & 297 & 2,6 & 35,0 & 2,9 & 41 & 2,4 & 2,6 \\
Styrax leprosus & 272 & 2,4 & 28,3 & 2,4 & 47 & 2,8 & 2,5 \\
Rudgea parquioides & 243 & 2,1 & 31,1 & 2,6 & 42 & 2,5 & 2,4 \\
Gymnanthes klotzschiana & 294 & 2,6 & 23,3 & 2,0 & 40 & 2,3 & 2,3 \\
Annona emarginata & 246 & 2,1 & 29,4 & 2,5 & 37 & 2,1 & 2,2 \\
Eugenia multicostata & 212 & 1,8 & 32,2 & 2,7 & 34 & 2,0 & 2,2 \\
Calyptranthes concinna & 258 & 2,2 & 23,9 & 2,0 & 39 & 2,3 & 2,2 \\
Eugenia uniflora & 221 & 1,9 & 27,8 & 2,3 & 31 & 1,8 & 2,0 \\
Araucaria angustifolia & 215 & 1,9 & 26,1 & 2,2 & 30 & 1,7 & 1,9 \\
Outras espécies & 2.999 & 26,0 & 402,8 & 33,9 & 450 & 26,3 & 28,7 \\
\hline Total & $\mathbf{1 1 . 5 3 8}$ & $\mathbf{1 0 0 , 0}$ & $\mathbf{1 . 1 8 7 , 8}$ & $\mathbf{1 0 0 , 0}$ & $\mathbf{1 . 7 1 2}$ & $\mathbf{1 0 0 , 0}$ & $\mathbf{1 0 0 , 0}$ \\
\hline
\end{tabular}


A distribuição de alturas da regeneração $($ Gini $=0,56)$ foi estatisticamente semelhante (Kolmogorov-Smirnov, $P<0,05)$ à distribuição de alturas da população das espécies Allophylus edulis (Gini =0,55) e Myrsine coriacea (Gini =0,54) (Figura 3). Contudo, a distribuição de alturas das espécies Casearia decandra $(\mathrm{Gini}=0,45)$, Cupania vernalis $(\mathrm{Gini}=0,61)$ e Matayba elaeagnoides $(\mathrm{Gini}=0,65)$ apresentaram diferenças significativas quando comparadas à distribuição geral da regeneração (Kolmogorov-Smirnov, $P<0,05$ ).

\section{Discussão}

A riqueza de espécies observada e estimada pelo Chao 2 indicou que foram amostradas mais de $85 \%$ da riqueza total de espécies da regeneração natural. Esse resultado evidencia que o esforço amostral adotado nesta pesquisa se mostrou satisfatório para representar a riqueza de espécies da área de estudo. Além disso, esses resultados se assemelharam ao encontrado em outros levantamentos realizados em Floresta Ombrófila Mista do Sul do Brasil, cuja faixa de variação está entre 39 e 109 espécies (CALDATO et al., 1996; BARDDAL et al., 2004; NARVAES et al., 2005; FIORENTIN et al., 2015; HIGUCHI et al., 2015).

Verificou-se que a diversidade de espécies do presente estudo foi superior a outros estudos sobre regeneração da Floresta Ombrófila Mista do Sul do Brasil (CALDATO et al., 1996; BARDDAL et al., 2004; NARVAES et al., 2005; BARBOSA et al., 2009;

FIGURA 3: Distribuição das frequências por classes de altura da regeneração natural e das cinco espécies mais importantes em uma Floresta Ombrófila Mista, Santa Catarina.
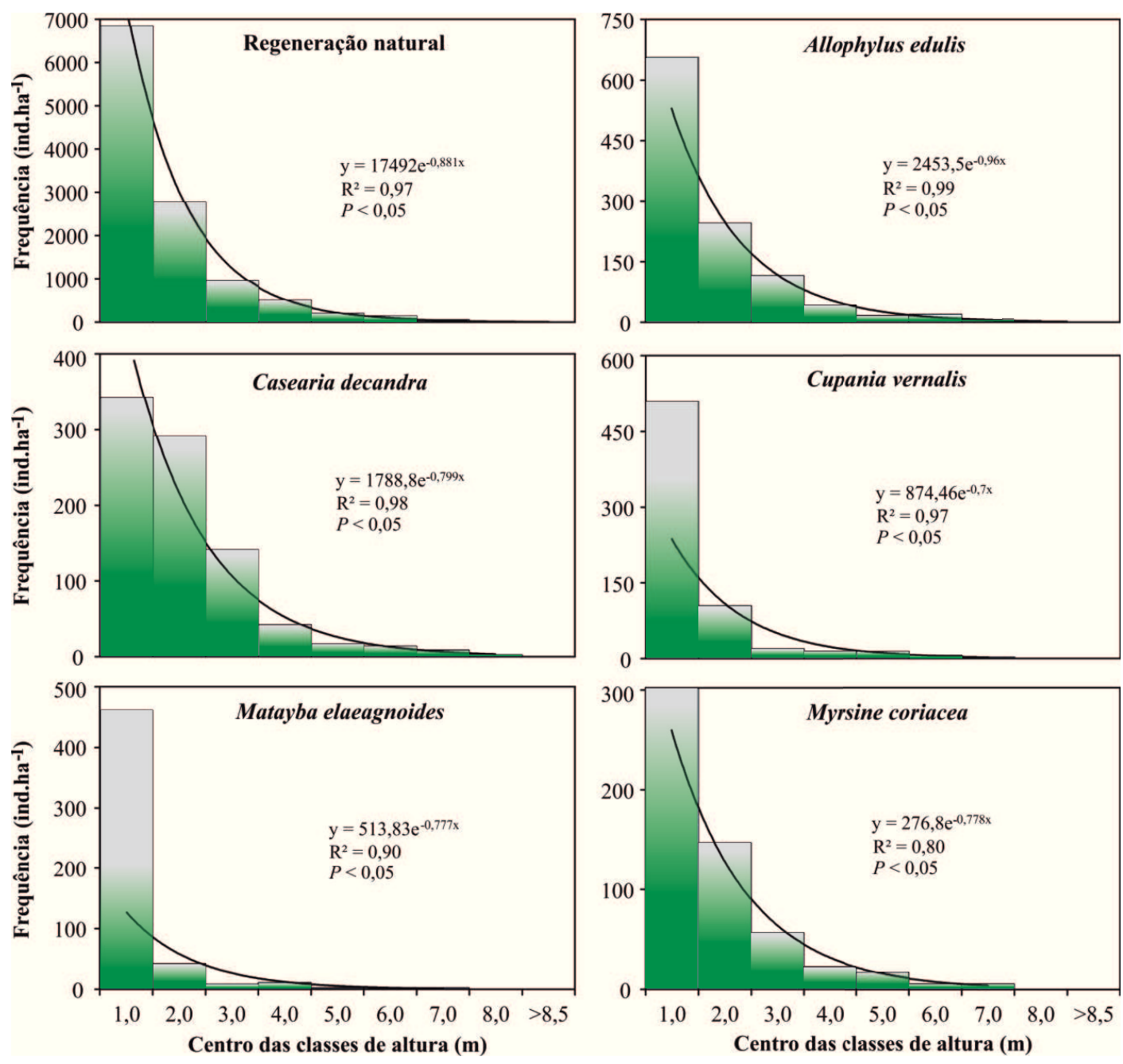
SANTOS et al., 2012; FIORENTIN et al., 2015), indicando que a área de estudo apresenta alto valor para conservação da diversidade biológica. Além disso, o valor do índice de Pielou demonstrou que, de modo geral, na área de estudo, a abundância de indivíduos entre as espécies está distribuída de forma equitativa (ver MAGURRAN, 2004), pois foi verificada a existência de poucas espécies altamente dominantes na amostra.

Algumas famílias registradas no presente estudo (Myrtaceae, Fabaceae, Euphorbiaceae, Sapindaceae e Solanaceae) são as com maior número de espécies da Floresta Ombrófila Mista de Santa Catarina, na qual as mais ricas são Myrtaceae, Lauraceae, Salicaceae e Sapindaceae (KLAUBERG et al., 2010; GASPER et al., 2013). O fato de Lauraceae não estar entre as famílias mais ricas na área de estudo sugere que a regeneração florestal encontra-se em fase de recomposição de sua riqueza, uma vez que a maioria das espécies dessa família é tolerante à sombra e ocorre nos diferentes estratos de florestas bem conservadas (BARBOSA et al., 2009; KLAUBERG et al., 2010; CHAMI et al., 2011). Além disso, verificou-se que as espécies clímax exigentes de luz foram as mais expressivas na área de estudo, evidenciando que o favorecimento dessas espécies pode ocorrer em função de fatores históricos como o uso anterior da área (AVILA et al., 2011; CHAMI et al., 2011), a ocorrência de perturbações naturais devido à abertura de clareiras pela queda de árvores ou, ainda, pela ação antrópica relacionada aos efeitos de borda da floresta (RIGUEIRA et al., 2012).

Apesar de as espécies mais importantes possuírem elevada abundância de indivíduos na regeneração, apenas algumas (Allophylus edulis, Casearia decandra, Cupania vernalis e Matayba elaeagnoides) apresentaram dispersão ampla pela área de estudo. Esse resultado evidencia que a maioria das espécies que apresentou elevada densidade de indivíduos, mas baixa frequência absoluta, pode estar distribuída de forma agrupada. O agrupamento dessas espécies pode estar relacionado com o favorecimento em relação à presença de microclimas diferenciados ou, ainda, ao surgimento de diferentes trajetórias sucessionais da regeneração natural que são influenciadas pela intensidade de distúrbios na área de estudo (CHAZDON, 2008). O resultado obtido quanto à distribuição da frequência das espécies é condizente com o padrão observado para a maioria das espécies em florestas tropicais e subtropicais que apresentam distribuição agregada (SILVA et al., 2012a). Vale destacar que $71,9 \%$ das espécies registradas apresentaram frequência inferior a $20 \%$, o que corresponde a apenas $18,5 \%$ da densidade total de indivíduos. De modo geral, o aumento da complexidade estrutural dessas florestas ocorre de maneira gradual, pois durante a trajetória sucessional existem poucas espécies dominantes e muitas espécies com poucos indivíduos (WIRTH et al., 2009).

A espécie mais importante da regeneração, Allophylus edulis, apresentou distribuição de alturas estatisticamente semelhante à distribuição geral da regeneração, evidenciando que sua população está encontrando ótimas condições naturais para o estabelecimento (KLEIN, 1980). Essa espécie é frequentemente mencionada como uma das mais importantes nos estudos sobre regeneração da Floresta Ombrófila Mista (CALDATO et al., 1996; BARDDAL et al., 2004; NARVAES et al., 2005; MEYER et al., 2013), principalmente pelo elevado número de indivíduos de menores tamanhos encontrados nos estratos médio e inferior da floresta. Por outro lado, três espécies, Casearia decandra, Cupania vernalis e Matayba elaeagnoides, apresentaram diferenças significativas quando comparadas à distribuição geral da regeneração, evidenciando que pode estar havendo um desequilíbrio entre as taxas de mortalidade e recrutamento dos indivíduos (NASCIMENTO et al., 2001). Essas espécies já foram apontadas como as mais importantes em estudos sobre regeneração natural (CALDATO et al., 1996; NARVAES et al., 2005; AVILA et al., 2011; CHAMI et al., 2011; VIBRANS et al., 2011) e no estrato arbóreo (NASCIMENTO et al., 2001; HIGUCHI et al., 2012) da Floresta Ombrófila Mista no Sul do Brasil. Ainda, essas espécies são classificadas como generalistas por demonstrarem elevada adaptabilidade a diversas condições ambientais, podendo ocorrer desde em florestas degradadas ou que sofreram perturbações antrópicas recentes até em florestas maduras e em vias adiantadas do processo de sucessão (NARVAES et al., 2005; VIBRANS et al., 2011). Por exemplo, em ambientes perturbados, essas 
espécies são muito comuns sob o dossel das araucárias, sendo representadas por elevado número de indivíduos regenerantes (KLAUBERG et al., 2010).

A distribuição de alturas encontrada para Myrsine coriacea foi estatisticamente semelhante ao padrão da distribuição geral da regeneração, evidenciando que sua população encontra-se em ótimas condições de regeneração na floresta. O fato de Myrsine coriacea aparecer com elevada densidade de indivíduos regenerantes na área de estudo pode estar relacionado com suas características ecológicas, uma vez que ela ocorre preferencialmente em áreas perturbadas e nas bordas das florestas (KLEIN, 1980; CARVALHO, 2003; MAÇANEIRO et al., 2015a). Além disso, sua elevada abundância pode estar relacionada com distúrbios naturais e antrópicos na área de estudo, pois ambos contribuem efetivamente nas mudanças estruturais das comunidades vegetais (CHAZDON, 2008). Apesar do mesmo nível de importância encontrado para Myrsine coriacea, a proporção de espécies (18\%) e de indivíduos $(13,4 \%)$ de pioneiras neste estudo foi inferior ao encontrado por Meyer et al. (2013). Esses autores registraram $25 \%$ de espécies e $30,7 \%$ dos indivíduos como pioneiros, o que denota que a floresta aqui estudada está mais bem preservada que a regeneração da Floresta Ombrófila Mista em Santa Catarina. Após 28 anos do fim da exploração florestal, a baixa participação das espécies pioneiras também evidencia que a regeneração florestal encontra-se em fase adiantada de reconstrução de sua estrutura.

Vale destacar a importância fisionômica que Araucaria angustifolia imprime nas formações da Floresta Ombrófila Mista do Sul do Brasil (KLEIN, 1960; KERSTEN et al., 2015), e que a regeneração florestal do presente estudo apresentou valores fitossociológicos medianos, mas bastante superiores aos valores encontrados por Meyer et al. (2013) na Floresta Ombrófila Mista do estado de Santa Catarina. Paludo et al. (2011) mencionaram que essa espécie apresenta uma fase crítica de regeneração dos indivíduos com até $50 \mathrm{~cm}$ de altura e que a mortalidade se estende até os 2 $\mathrm{m}$ de altura, mas, como é uma espécie longeva, ela pode manter um baixo número de indivíduos regenerantes na população e, mesmo assim, apresentar sucesso na regeneração natural.
A área estudada revelou valores de riqueza e diversidade elevados para a fitofisionomia em questão, fato corroborado pelo índice de Shannon, um dos maiores valores já registrados para a regeneração da Floresta Ombrófila Mista do Sul do Brasil. Os elevados valores de densidade, frequência e categoria de tamanho apontaram Allophylus edulis, Casearia decandra, Cupania vernalis, Matayba elaeagnoides e Myrsine coriacea como as espécies mais importantes da regeneração florestal na área de estudo, as quais potencialmente poderão compor parte do estrato arbóreo da floresta no futuro. A semelhança entre a distribuição de alturas de Allophylus edulis e Myrsine coriacea com a distribuição geral da regeneração evidenciou que a população dessas espécies está encontrando condições adequadas de regeneração. Por fim, a participação de espécies pioneiras na regeneração foi relativamente baixa, fato que, aliado às demais conclusões apontadas acima, indica que a regeneração encontra-se em fase avançada de reconstrução de sua composição e estrutura.

\section{Referências}

APG IV. An update of the Angiosperm Phylogeny Group classification for the orders and families of flowering plants: APG IV. Botanical Journal of the Linnean Society, London, v. 181, n. 1, p. 1-20, 2016.

AVILA, A. L.; ARAUJO, M. M.; LONGHI, S. J.; GASPARIN, E. Agrupamentos florísticos na regeneração natural em remanescentes de Floresta Ombrófila Mista, RS, Brasil. Scientia Forestalis, Piracicaba, v. 39, n. 91, p. 331-342, 2011.

BARBOSA, C. E. A.; BENATO, T.; CAVAlHEIRO, A. L.; TOREZAN, J. M. Diversity of regenerating plants in reforestations with Araucaria angustifolia (Bertol.) O. Kuntze of 12, 22, 35, and 43 years of age in Paraná State, Brazil. Restoration Ecology, Crawley, v. 17, n. 1, p. 60-67, 2009.

BARDDAL, M. L.; RODERJAN, C. V.; GALVÃO, F.; CURCIO, G. R. Fitossociologia do sub-bosque de uma Floresta Ombrófila Mista Aluvial, no município de Araucária, PR. Ciência Florestal, Santa Maria, v. 14, n. 1, p. 35-45, 2004.

CALDATO, S. L.; FLOSS, P. A.; CROCE, D. M.; LONGHI, S. J. Estudo da regeneração natural, banco de sementes e chuva de sementes na Reserva Genética Florestal de Caçador, SC. Ciência Florestal, Santa Maria, v. 6, n. 1, p. 27-38, 1996.

CARVALHO, P. E. R. Espécies arbóreas brasileiras. Colombo: Embrapa Florestas, 2003. 1.039 p.

CHAMI, L. B.; ARAUJO, M. M.; LONGHI, S. J.; KIELSE, P.; DAL'COL LÚCIO, A. Mecanismos de regeneração natural em diferentes ambientes de remanescentes de Floresta Ombrófila Mista, São Francisco de Paula, RS. Ciência Rural, Santa Maria, v. 41, n. 2, p. 251-259, 2011. 
CHAZDON, R. L. Chance and determinism in tropical forest succession. In: CARSON, W.; SCHNITZER, S. A. (Ed.). Tropical forest community ecology. Oxford: Wiley-Blackwell Publishing, 2008. p. 384-408.

CHAZDON, R. L. Regeneração de florestas tropicais. Boletim do Museu Paraense Emílio Goeldi. Ciências Naturais, Belém, v. 7, n. 3, p. 195-218, 2012.

DENT, D. H.; WRIGHT, S. J. The future of tropical species in secondary forests: a quantitative review. Biological Conservation, Boston, v. 142, n. 12, p. 2833-2843, 2009.

EMBRAPA. Solos do estado de Santa Catarina. Rio de Janeiro: Embrapa Solos, 2004. 745 p.

EPAGRI. Atlas climatológico do estado de Santa Catarina. Florianópolis: EPAGRI, 2002. CD 1. Versão eletrônica.

FELFILI, J. M. Dynamics of the natural regeneration in the Gama gallery forest in Central Brazil. Forest Ecology and Management, Amsterdam, v. 91, n. 2-3, p. 235-245, 1997.

FIORENTIN, L. D.; TÉO, S. J.; SCHNEIDER, C. R.; COSTA, R. H.; BATISTA, S. Análise florística e padrão espacial da regeneração natural em área de Floresta Ombrófila Mista na região de Caçador, SC. Floresta e Ambiente, Seropédica, v. 22, n. 1, p. 60-70, 2015.

FLORA DO BRASIL. Flora do Brasil 2020 em construção. Jardim Botânico do Rio de Janeiro. Disponível em <http://floradobrasil. jbrj.gov.br/>. Acesso em: 07 junho 2016.

GASPER, A. L.; SEVEGNANI, L.; VIBRANS, A. C.; SOBRAL, M.; UHLMANN, A.; LINGNER, D. V.; RIGON-JÚNIOR, M. J.; VERDI, M.; STIVAL-SANTOS, A.; DREVECK, S.; KORTE, A. Inventário florístico florestal de Santa Catarina: espécies da Floresta Ombrófila Mista. Rodriguésia, Rio de Janeiro, v. 64, n. 2 , p. 201-210, 2013.

HIGUCHI, P.; SILVA, A. C.; BUZZI JUNIOR, F.; NEGRINII, M.; FERREIRA, T. S.; SOUZA, S. T.; SANTOS, K. F.; VEFAGO, M. B. Fatores determinantes da regeneração natural em um fragmento de floresta com araucária no planalto catarinense. Scientia Forestalis, Piracicaba, v. 43, n. 106, p. 251-259, 2015.

HIGUCHI, P.; SILVA, A. C.; FERREIRA, T. S.; SOUZA, S. T.; GOMES, J. P.; SILVA, K. M.; SANTOS, K. F.; LINKE, C.; PAULINO, P. S. Influência de variáveis ambientais sobre o padrão estrutural e florístico do componente arbóreo, em um fragmento de Floresta Ombrófila Mista Montana em Lages, SC. Ciência Florestal, Santa Maria, v. 22, n. 1, p. 79-90, 2012.

HOSOKAWA, R. T.; MOURA, J. B.; CUNHA, U. S. Introdução ao manejo e economia de florestas. Curitiba: Editora UFPR, 2008. $164 \mathrm{p}$.

IBGE. Manual Técnico da Vegetação Brasileira. Rio de Janeiro: Instituto Brasileiro de Geografia e Estatística, 2012. 271p.

KERSTEN, R. A.; BORGO, M.; GALVÃO, F. Floresta Ombrófila Mista: aspectos fitogeográficos, ecológicos e métodos de estudo. In: EISENLOHR, P. V.; FELFILI, J. M.; MELO, M. M. R. F.; ANDRADE, L. A.; MEIRA NETO, J. A. A. (Ed.). Fitossociologia no Brasil: métodos e estudos de casos. Viçosa: Editora UFV, 2015. p. 156-182.

KlauberG, C.; PAludo, G. F.; BORTOluZZI, R. L. C.; MANTOVANI, A. Florística e estrutura de um fragmento de Floresta Ombrófila Mista no Planalto Catarinense. Biotemas, Florianópolis, v. 23, n. 1, p. 35-47, 2010.
KLEIN, R. M. O aspeto dinâmico do pinheiro brasileiro. Sellowia, Itajaí, v. 12, p. 17-44, 1960.

KLEIN, R. M. Ecologia da flora e vegetação do Vale do Itajaí. Sellowia, Itajaí, v. 32, 165-389, 1980.

LEITE, P. F. Contribuição ao conhecimento fitoecológico do sul do Brasil. Ciência \& Ambiente, Santa Maria, v. 13, n. 24, p. 51-73, 2002.

MAÇANEIRO, J. P.; SCHORN, L. A.; SEVEGNANI, L.; VIBRANS, A. C. Structure of the tree component and indicator species in different types of forests in the Itajaí-Mirim river, Southern Brazil. Australian Journal of Basic and Applied Sciences, Jordan, v. 9, n. 33, p. 392-397, 2015a.

MAÇANEIRO, J. P.; SEUBERT, R. C.; SCHORN, L. A. Fitossociologia de uma floresta pluvial subtropical primária no sul do Brasil. Floresta, Curitiba, v. 45, n. 3, p. 555-566, 2015 b.

MAGURRAN, A. E. Measuring biological diversity. Malden: Blackwell Publishing, 2004. 256 p.

MEYER, L.; GASPER, A. L.; SEVEGNANI, L.; SCHORN, L. A.; VIBRANS, A. C.; LINGNER, D. V.; VERDI, M.; STIVALSANTOS, A.; DREVECK, S.; KORTE, A.. Regeneração natural da Floresta Ombrófila Mista em Santa Catarina. In: VIBRANS, A. C.; SEVEGNANI, L.; GASPER, A. L.; LINGNER, D. V. (Ed.). Inventário florístico florestal de Santa Catarina: Floresta Ombrófila Mista. Blumenau: Edifurb, 2013. p. 191-222.

NARVAES, I. S.; BRENA, D. A.; LONGHI, S. J. Estrutura da regeneração natural em floresta ombrófila mista na Floresta Nacional de São Francisco de Paula, RS. Ciência Florestal, Santa Maria, v. 15, n. 4, p. 331-342, 2005.

NASCIMENTO, A. R. T.; LONGHI, S. J.; BRENA, D. A. Estrutura e padrões de distribuição espacial de espécies arbóreas em uma amostra de floresta ombrófila mista em Nova Prata, RS. Ciência Florestal, Santa Maria, v. 11, n. 1, p. 105-119, 2001.

OLIVEIRA-FILHO, A. T. Estudos ecológicos da vegetação como subsídios para programas de revegetação com espécies nativas: uma proposta metodológica. Cerne, Lavras, v. 1, n. 1, p. 64-72, 1994.

PALUDO, G. F.; MANTOVANI, A.; REIS, M. S. Regeneração de uma população natural de Araucaria angustifolia (Araucariaceae). Revista Árvore, Viçosa, v. 35, n. 5, p. 1107-1119, 2011.

PAN, Y.; BIRDSEY, R. A.; FANG, J.; HOUGHTON, R.; KAUPPI, P. E.; KURZ, W. A.; PHILLIPS, O. L.; SHVIDENKO, A.; LEWIS, S. L.; CANADELL, J. G.; CIAIS, P.; JACKSON, R. B.; PACALA, S. W.; MCGUIRE, A. D.; PIAO, S.; RAUTIAINEN, A.; SITCH, S.; HAYES, D. A large and persistent carbon sink in the world's forests. Science, New York, v. 333, n. 6045, p. 988-993, 2011.

RIGUEIRA, D. M. G.; MOLINARI, A. L. M.; MARIANO, D. L. S.; REIS, R. M.; PORTUGAL, A. B.; SANTANA, N. S.; SANTOS, R. A. Influência da distância da borda e do adensamento foliar sobre a abundância de plantas pioneiras em um fragmento de floresta tropical submontana na Estação Ecológica de Wenceslau Guimarães (Bahia, Brasil). Acta Botanica Brasilica, Feira de Santana, v. 26, n. 1, p. 197-202, 2012.

SANTA CATARINA. Atlas de Santa Catarina. Florianópolis: GAPLAN/SUEGI, 1986. 173 p.

SANTOS, S. C.; BUDKE, J. C.; MULLER, A. Regeneração de espécies arbóreas sob a influência de Merostachys multiramea Hack. (Poaceae) em uma floresta subtropical. Acta Botanica Brasilica, Feira de Santana, v. 26, n. 1, p. 218-229, 2012. 
SEVEGNANI, L.; UHLMANN, A.; GASPER, A. L.; VIBRANS, A. C.; STIVAL-SANTOS, A.; VERDI, M.; DREVECK, S. Estádios sucessionais da Floresta Ombrófila Mista em Santa Catarina. In: VIBRANS, A. C.; SEVEGNANI, L.; GASPER, A. L.; LINGNER, D. V. (Ed.). Inventário florístico florestal de Santa Catarina: Floresta Ombrófila Mista. Blumenau: Edifurb, 2013. p. 255-271.

SIEGEL, S.; CASTELLAN, N. J. Estatística não-paramétrica para as ciências do comportamento. Porto Alegre: Artmed, 2006. $448 \mathrm{p}$.

SILVA, A. C.; HIGUCHI, P.; AGUIAR, M. D.; NEGRINI, M.; FERT NETO, J.; HESS, A. F. Relações florísticas e fitossociológicas de uma Floresta Ombrófila Mista Montana secundária em Lages, Santa Catarina. Ciência Florestal, Santa Maria, v. 22, n. 1, p. 193206, 2012b.

SILVA, A. C.; HIGUCHI, P.; NEGRINI, M.; GRUDTNER, A.; ZECH, D. F. Caracterização fitossociológica e fitogeográfica de um trecho de floresta ciliar em Alfredo Wagner, SC, como subsídio para restauração ecológica. Ciência Florestal, Santa Maria, v. 23, n. 4, p. 579-593, 2013.

SILVA, K. E.; MARTINS, S. V.; SANTOS, N. T.; RIBEIRO, C. A. A. S. Padrões espaciais de espécies arbóreas tropicais. In: MARTINS, S. V. (Ed.). Ecologia de florestas tropicais do Brasil. Viçosa: Editora UFV, 2012a. p. 326-352.

SIMINSKI, A.; FANTINI, A. C.; REIS, M. S. Classificação da vegetação secundária em estágios de regeneração da Mata Atlântica em Santa Catarina. Ciência Florestal, Santa Maria, v. 23, n. 3, p. 369-378, 2013.
SWAINE, M. D.; WHITMORE, T. C. On the definition of ecological species groups in tropical rain forests. Vegetatio, Perth, v. 75, n. 1, p. 81-86, 1988.

THOMAS, W. W.; CARVALHO, A. M. V.; AMORIM, A. M. A.; GARRISON, J.; ARBELÁEZ, A. L. Plant endemism in two forests in southern Bahia, Brasil. Biodiversity and Conservation, New York, v. 7, n. 3, p. 311-322, 1998.

VIBRANS, A. C.; McROBERTS, R. E.; MOSER, P.; NICOLETTI, A. L. Using satellite image-based maps and ground inventory data to estimate the area of the remaining Atlantic forest in the Brazilian state of Santa Catarina. Remote Sensing of Environment, Elsevier, v. 130, n. 3, p. 87-95, 2013.

VIBRANS, A. C.; SEVEGNANI, L.; UHLMANN, A.; SCHORN, L. A.; SOBRAL, M. G.; GASPER, A. L.; LINGNER, D. V.; BROGNI, E.; KLEMZ, G.; GODOY, M. B.; VERDI, M. Structure of Mixed Ombrophyllous Forests with Araucaria angustifolia (Araucariaceae) under external stress in southern Brazil. Revista de Biología Tropical, San José, v. 59, n. 3, p. 1371-1387, 2011.

WEINER, J.; SOLBRIG, O. T. The meaning and measurement of size hierarchies in plant populations. Oecologia, Heidelberg, v. 61, n. 3, p. 334-336, 1984.

WIRTH, C.; MESSIER, C.; BERGERON, Y.; FRANK, D.; FANKHÄNEL, A. Old-growth forest definitions: a pragmatic view. In: WIRTH, C.; GLEIXNER, G.; HEIMANN, M. (Ed.). Old-growth forests: function, fate and value. New York: Springer, 2009. p. 11-33. 